EPJ Web of Conferences 19, 08005 (2012)

DOI: $10.1051 /$ epjconf/20121908005

(C) Owned by the authors, published by EDP Sciences, 2012

\title{
Chemical homogeneity in the Orion Association: Oxygen abundances of B stars
}

\author{
K. Cunha ${ }^{1,2, a}$, I. Hubeny ${ }^{2}$ and T. Lanz ${ }^{3}$ \\ ${ }^{1}$ Observatorio Nacional, Rio de Janeiro, Brazil \\ ${ }^{2}$ University of Arizona, Steward Observatory, USA \\ ${ }^{3}$ University of Maryland, USA
}

\begin{abstract}
We present non-LTE oxygen abundances for a sample of B stars in the Orion association. The abundance calculations included non-LTE line formation and used fully blanketed non-LTE model atmospheres. The stellar parameters were the same as adopted in the previous study by Cunha \& Lambert (1994). We find that the young Orion stars in this sample of 10 stars are described by a single oxygen abundance with an average value of $\mathrm{A}(\mathrm{O})=8.78$ and a small dispersion of \pm 0.05 , dex which is of the order of the uncertainties in the analysis. This average oxygen abundance compares well with the average oxygen abundance obtained previously in Cunha \& Lambert (1994): $\mathrm{A}(\mathrm{O})=8.72 \pm 0.13$ although this earlier study, based upon non-blanketed model atmospheres in LTE, displayed larger scatter. Small scatter of chemical abundances in Orion B stars had also been found in our previous studies for neon and argon; all based on the same effective temperature scale. The derived oxygen abundance distribution for the Orion association compares well with other results for the oxygen abundance in the solar neighborhood.
\end{abstract}

\section{INTRODUCTION}

Oxygen is one of the most abundant elements and is produced mainly in hydrostatic burning in massive stars and ejected in the interstellar medium via Type II SNe. It tracks star formation and massive star evolution and is recycled very quickly into the interstellar medium. Modelling of the stellar atmospheres of early type stars has advanced in recent years with improvements in the atomic data making full self-consistent non-LTE calculations possible. In this contribution we present oxygen abundances for a sample of young stars in the Orion association.

\section{ANALYSIS AND RESULTS}

The target stars are $10 \mathrm{OB}$ main-sequence stars members of the subgroups of the Orion association. Full non-LTE calculations were done using non-LTE model atmospheres computed by TLUSTY [3] with updated and extended model atoms. The stellar parameters were the same as adopted in the previous study of [1]. Oxygen abundances were obtained from the best fits between observed and synthetic spectra from a large number of O II transitions.

The average oxygen abundance obtained for the studied sample is: $\mathrm{A}(\mathrm{O})=8.78 \pm 0.05$ dex. The scatter in the derived $\mathrm{A}(\mathrm{O})$ can be completely explained in terms of the abundance uncertainties in the analysis. In Figure 1 we show a comparison of the oxygen abundances obtained here (top panel) with other recent studies from the literature. The middle panel shows results from [6] and [7] that were

ae-mail: katia@on.br

This is an Open Access article distributed under the terms of the Creative Commons Attribution-Noncommercial License 3.0, which permits unrestricted use, distribution, and reproduction in any noncommercial medium, provided the original work is properly cited. 


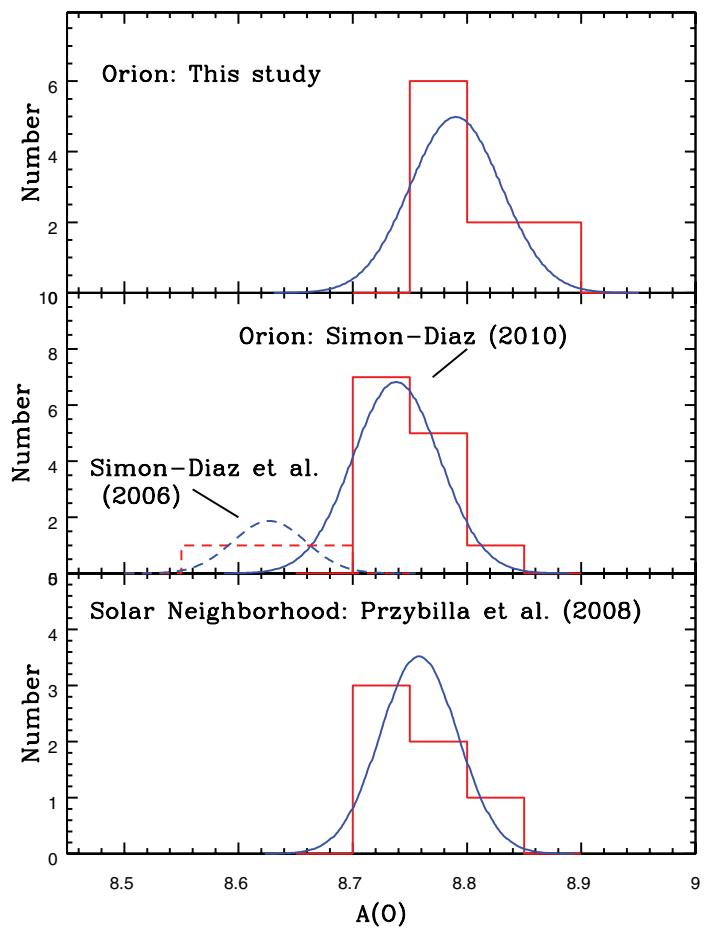

Figure 1. A comparison of our results with other recent results from the literature for the Orion association (middle panel) and for the solar neighborhood (bottom panel). The oxygen abundances overall compare well. Indications that the Orion association chemical composition is homogeneous had been found in our previous studies for neon [2] and argon [4] all based on the same effective temperature scale. Recent results from [8] also confirm this homogeneity.

obtained with FASTWIND models. Our abundances overlap the most recent study from this group but not the lower oxygen from [6]. For comparison, we also show in the bottom panel the results from [5] for a sample of 6 stars in the solar neighborhood: the solar vicinity abundances and scatter compare well with our results for Orion. Taken together these results indicate that the chemical abundances in the Orion association are homogeneous and agree well with solar neighborhood values. The earlier oxygen abundance distribution from [1] presented more scatter, although with the same average oxygen abundance.

\section{References}

[1] Cunha, K. \& Lambert D. L., ApJ, 426, (1994) 170

[2] Cunha, K., Hubeny, I. \& Lanz, T., ApJL 647, (2006) 143

[3] Hubeny, I. \& Lanz, T., ApJ 439, (1995) 875

[4] Lanz, T. Cunha, K., Holtzman, J., \& Hubeny, I. 678, (2008) 1342

[5] Przybilla, N., Nieva, M., \& Butler, K. ApJL 688, (2008) 103

[6] Simon-Diaz, S., Herrero, A., Esteban, C., \& Najarro, A\&A 448, (2006) 351

[7] Simon-Diaz, S., A\&A 510, (2010) 22

[8] Nieva, M.-F.; Simon-Diaz, S., A\&A, 532, (2011) 2 